\title{
Second Intervention in Obstetric Hemorrhage
}

\author{
Rawal S. , Rana A. \\ Department of Obstetrics and Gynecology, Tribhuvan University Teaching Hospital, Kathmandu, Nepal \\ Correspondence address: Dr. Suniti Rawal, Department of Obstetrics and Gynecology \\ Tribhuvan University Teaching Hospital \\ E-mail: sunudinurawal@yahoo.com
}

\begin{abstract}
Introduction: Obstetric hemorrhage is still one of the dreaded complications that contribute to a maximum number of maternal morbidity and mortality till date. The management of obstetric hemorrhage involves early recognition, assessment and resuscitation. Though dealt with appropriate use of oxytocic agents, it may seldom require surgical techniques, including uterine tamponade, major vessel ligation, compression sutures, and even hysterectomy.
\end{abstract}

Method: Prospective study of 20 cases of laparotomy for obstetrical hemorrhage carried out at Tribhuvan University Teaching Hospital, Kathmandu, Nepal, between Jan 2003 to Nov 2011.

Results: Out of 20 cases, massive hemoperitoneum (more than a liter) was noted in 9 and associated risk factors in 10 . Source of bleeding in 20 cases were from extensive hematoma (retroperitoneal and broad ligament) in 5 , including a rectus sheath hematoma and with colporrhexis, oozing inverted $\mathrm{T}$ incision repaired in a single layer (1), placental bed (3) and 1 was from vessels in LUS. There was bleeding from uterine angle (4) and incision (1). Bleeding from tear at various sites were 3 , from uterovesicle fold of peritoneum 1 and from the ruptured uterus following vacuum delivery in a case of VBAC (1). Uterine packing was done in 1, B-Lynch in 3 and 1 failed needing the uterine packing; uterine artery ligation in 2 including ovarian vessel ligation in 1, repair of ruptured uterus in 1 and subtotal hysterectomy in 5 cases. There were 3 mortalities due to DIC, pulmonary edema and ARF and rest were discharged in good health.

Conclusion: Choosing of the right technique, complete hemostasis and meticulous closure of all surgical incisions will prevent the need for laparotomy following LSCS. Vigilant monitoring of all the post operative patients will lead to early diagnosis of intraperitoneal / pervaginal bleeding and its management, thus preventing morbidity and mortality owing to late diagnosis.

Key Words: Intraperitoneal bleeding, hemoperitoneum, relaparotomy

\section{Introduction}

Obstetric hemorrhage is stillone of the dreaded complications that contribute to a maximum number of maternal morbidity and mortality till date. The management of obstetric hemorrhage involves early recognition, assessment and resuscitation. Though dealt with appropriate use of oxytocic agents, it may seldom require surgical techniques, including uterine tamponade, major vessel ligation, compression sutures, and even hysterectomy. Having the abdomen opened once and requiring a second intervention as for uterine pack removal in postoperative hemorrhage or repair following the primary defect persisting in the uterine wall after caesarean section or the application of B-lynch compression suture is better described as "relaparotomy" or in other word, second intervention that works as a remedy saving lives of many mothers ${ }^{1,2}$. The successful treatment of obstetric hemorrhage is still a challenge for both the developed and developing worlds.

The study concentrates on laparotomy as second interventions for hemorrhagic complications arising from 
obstetric surgery aiming to find out our constraints to convene a remedy and to reduce the number of maternal morbidity and mortality.

\section{Methods}

Prospective ongoing study to analyze cases of laparotomy done as second intervention following hemorrhage after Lower Segment Caesarean Section (LSCS), in the Department of Obstetrics and Gynecology, Tribhuvan University Teaching Hospital (TUTH), Kathmandu, Nepal, from Jan 2003 to Nov 2011. A total of 20 cases have been evaluated.

\section{Inclusion criteria}

The cases reopened following obstetric hemorrhage after caesarean section (CS)

\section{Exclusion criteria}

The cases that had relaparotomy for sepsis, paralytic ileus and burst abdomen due to infections were excluded.

\section{Results}

Over these years there were 20 Obstetrics cases that required second intervention/relaparotomy for the hemorrhagic complications that were collated for the evaluation of the defects.

The indications for performing the caesarean section were varied and the most common being the fetal distress (5), followed by 6 cases of previous caesarean section, for previous CS (1) VBAC in case of previous CS for breech who had a vacuum delivery for fetal distress in second stage of labour (1), with breech (1), obstetric cholestasis (1), rising antibody titer in Rh negative G4 P3 with previous 2 CS (1), and marginal placenta previa (1). Two were placenta previa including the one with previous caesarean section and eclampsia (1) with impending features (1). Non progress of labour, non descent of head, prolong 2nd stage of labour, obstructed labour with hand prolapse of the second twin made 4 cases and one each of primi breech and CPD.

Three referred cases did contribute to the bulk. The first referred case was primary CS performed for fetal distress. The patient had gradual progression of abdominal distention on the first post operative day with no urinary output and rising pallor. There was a huge right sided broad ligament hematoma measuring about $8 \times 10 \mathrm{~cm}$, which extended towards the lumbar region forming a $15 \times 15$ $\mathrm{cm}$ hematoma, uterus was flabby about 14 weeks size. She was reopened after 48 hours, had subtotal hysterectomy but succumbed to death due to DIC and ARF.

The second referred case was of primary CS with the repair of uterus for obstructed labour for 2nd twin with hand prolapsed with rupture uterus. She attended the emergency room on the $3 \mathrm{rd}$ postoperative day with shortness of breath, fever, abdominal distention for 2 days, pallor, edema, tachycardia and full flanks. USG showed haemoperitoneum. About $2500 \mathrm{ml}$ of blood mixed ascitic fluid was present on laparotomy. LSCS scar was intact. Sloughed, necrotic, infected tissue was present at the left uterine and vaginal wall extending to the left broad ligament, multiple catgut sutures were seen in these area which were confused with $\mathrm{u}$ possible rupture or extension of the uterine incision. A rent of $1.5 \times 2 \mathrm{~cm}$ was present at the left lateral uterine wall extending to the vagina. Left uterine artery was bisected with few catgut sutures on it overlying the left distended ureter. Left ureter was distended with few catgut sutures near the vesicouterine junction and the proximal ureter was distended. She underwent subtotal hysterectomy with the release of the catgut knots, ureter was decompressed and methylene blue dye was pushed retrograde via foley's catheter with no leakage. Later she developed ureteric fistula for which stenting was done and discharged home in good health. (Fig 1a, 1b, 1c)

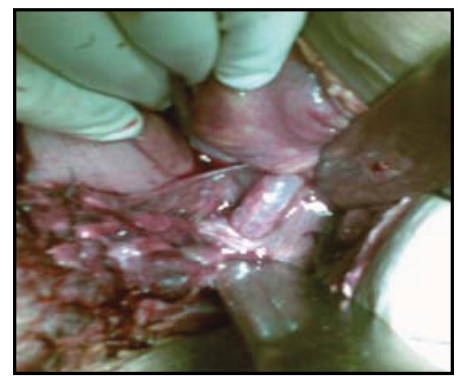

Fig. 1(a): Small bulge indicating the tied up ureter on the left side

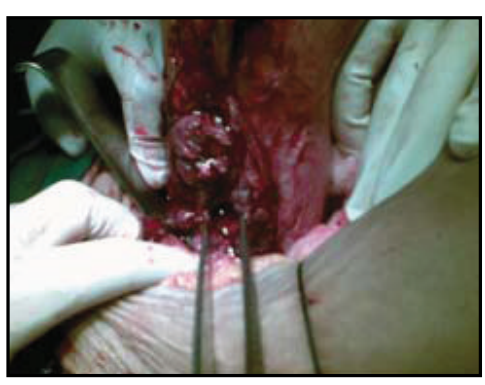

Fig. 1(b): Rent about 2x2 cm, near the left uterine artery and extending to left uterine angle 


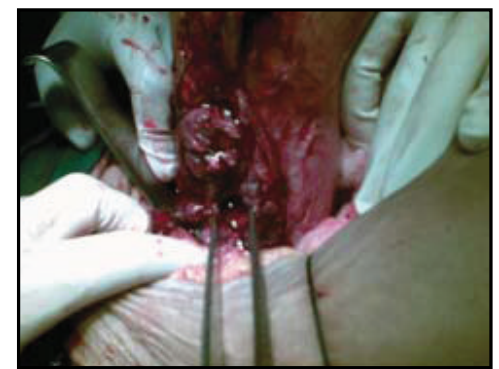

Fig. 1(c): Lateral view of the uterus after subtotal hysterectomy

The third case was also a referred case from Bhairahawa, who presented on the 2 nd post operative day following CS done for non progress of labour at $40+$ weeks and was referred to us for decreased urine output. On arrival at night, she was conscious and oriented but looked very pale. She also had abdominal distention with fluid thrill and shifting dullness. The pulse and blood pressure was maintained due to large amount of fluid that she has received. Urine output was minimal, about $200 \mathrm{ml}$ in the urobag. The investigations done at TUTH showed the haemoglobin of $6 \mathrm{mg} \%$, platelet count of $32,000 /$ cumm and ultrasonography showed haemoperitoneum. Time was taken for the arrangement of fresh blood and PRP and on reopening her after about 16 hours of arrival, hemoperitoneum of more than a liter with multiple oozers in the left angle of the lower uterine segment was seen. Bluish discoloration of peritoneum in the region of the $\mathrm{B} / \mathrm{L}$ broad ligament and vesicouterine fold of peritoneum was also observed. Oozers and the left angle were secured again. Proper haemostsis was maintained and after receiving multiple pints of fresh blood and PRP she was kept in ICU for more than a month. But despite all the effort she succumbed to death due to complications like pulmonary oedema and multi organ failure.

\section{Symptoms and sign on presentation:}

Most of these patients had rising pallor with tachycardia ranging from 100-140 beats/ min, hypotension, sweating, dizziness, abdominal distention and decrease in urinary output. Four cases had uterine atony. Some of them had increase pervaginal bleeding in the operating table during vaginal toileting or in the post operative ward after being transferred.

USG was of intense help to diagnose haemoperitoneum in many cases, which made the diagnosis prompt, easy and second intervention much quicker. Fig 1 (d)

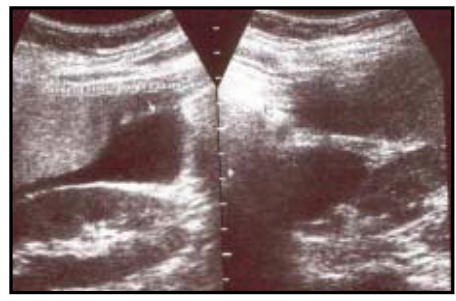

Fig. 1 (d): USG showing fluid collection in Morrison's pouch and peritoneal cavity

\section{Time taken for second intervention}

The time taken for second intervention following primary $\mathrm{CS}$ varied from 15 minutes in the operation room where excessive bleeding was noticed at the time of vaginal toileting at the end of surgery or up to 3 days where abdominal distension was observed along with decreasing output and increasing pallor. [Within an hour (3); 3-4 (2), 4-7 h (2), 8-12 h (4), 13- 24 h (4), 25-48 hours (4), 48-72 hrs (1)]

\section{At laparotomy}

Significant intraperitoneal hemoperitoneum of more than $2 \mathrm{~L}$ was noted in a case where primary CS was done for obstructed labour of 2 nd twin with hand prolapse with rupture uterus along with the repair of uterus. The amount of blood loss was more than $2.5 \mathrm{~L}$. The other 8 cases had loss of about 1 to $1.5 \mathrm{~L}$. Two of the case, who had primary CS done for CPD and NPOL respectively had blood loss of about a liter but both succumbed to death following massive haematoma and DIC in the first case and pulmonary oedema with multi organ failure in the second. Fig 2 (a)

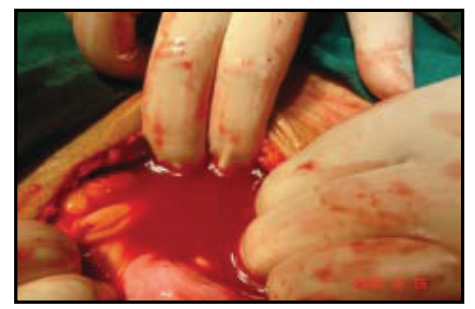

Fig. 2 (a): Haemoperitoneum

There were 5 cases with extensive hematoma among which one had colporrhexis of about $4 \mathrm{~cm}$ and the other with rectus sheath hematoma. A case as mentioned earlier developed broad ligament hematoma of $15 \times 15 \mathrm{~cm}$ extending to the lumbar region, had subtotal hysterectomy but died due to DIC and ARF.

There were cases with oozing inverted $\mathrm{T}$ incision repaired 
in a single layer (1); placental bed (3) and 1 was from vessels in lower uterine segment. There was bleeding from uterine angle (4) and incision (1).A patient had bleeding from uterovesicle fold of peritoneum and three patients bleed from tear at various sites (Fig 2b, 2c). An unbooked case who previously had a CS for Primi breech in the valley arrived to labour room in second stage of labour with thick meconium and fetal bradycardia. Vacuum was applied with a good outcome but later complained of pain abdomen, and giddiness. She had tachycardia and low blood pressure and USG showed haemoperitoneum. She was reopened after about $8 \mathrm{hrs}$ to find ruptured uterus with hemoperitoneum of $1000 \mathrm{ml}$. There was an inverted T shaped rupture uterus with a gap both in the lower as well as the upper segment making a " $T$ " of about $3 \mathrm{~cm}$ which was repaired in 2 layers securing the hemostasis followed by $\mathrm{B} / \mathrm{L}$ tubal ligation.

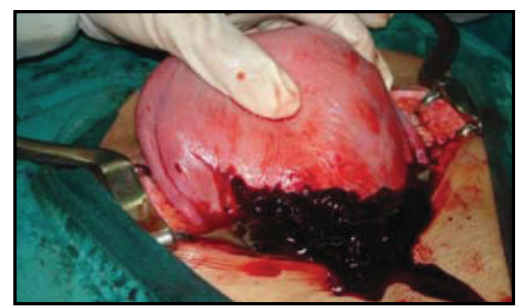

Fig. 2(b): Blood and clot collection at the incision line

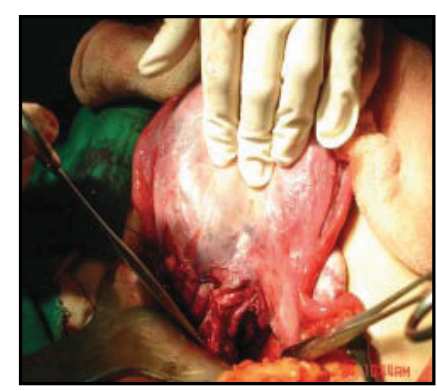

Fig. 2(c): Tear being reinforced

\section{Second intervention and repair done}

The treatment were carried out by evacuating the hematoma and suturing the vaginal rent (1), securing the angles (4) and the bleeder from the uterovesicle fold of peritoneum (1), reopening the loosely stitched lower uterine segment (2) and resuturing it. Oozing and repair of the inverted $\mathrm{T}$ shaped was reinforced (2) (Fig 3a), applying multiple stitches in the placental bed (3). Tear extending from the uterine angles was repaired in (3). (Fig 3b)

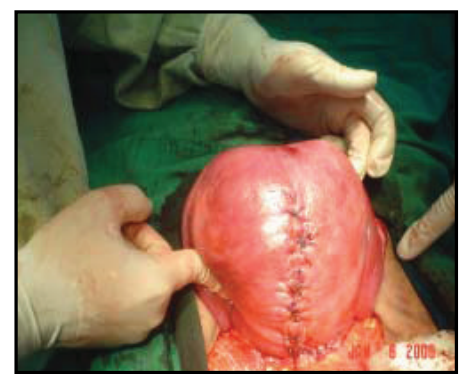

Fig. 3 (a): Inverted $\mathrm{T}$ incision after being reinforced

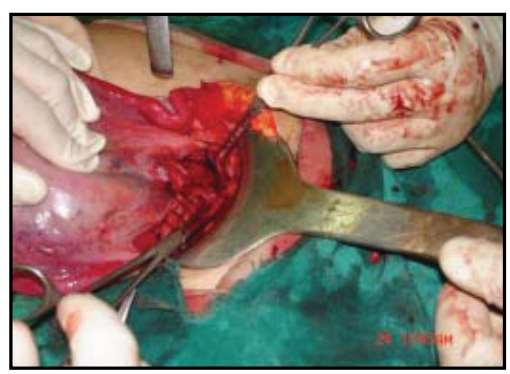

Fig. 3(b): Repair of the extension of uterine angle

Uterine vessels were ligated in 2 cases including ovarian pedicle ligation in (1). Uterus was packed in a case where bilateral uterine as well as ovarian vessels were ligated; however, B-lynch suture was not of much help too (Fig 4a). The uterine pack was removed after $36 \mathrm{hrs}$ with no further pervaginal bleeding. B-lynch suture was applied (Fig 4 b) in 3 cases including the one stated above. Subtotal hysterectomy was performed in 5 cases (Fig 5), particularly for extensive hematomas (2), massive bleeding from the placental bed (1), generalized oozing from bilateral angles (1), previous rent of the ruptured uterus (1), with good result except in 3 cases where mortality resulted from DIC, ARF, pulmonary oedema and multiorgan failure.

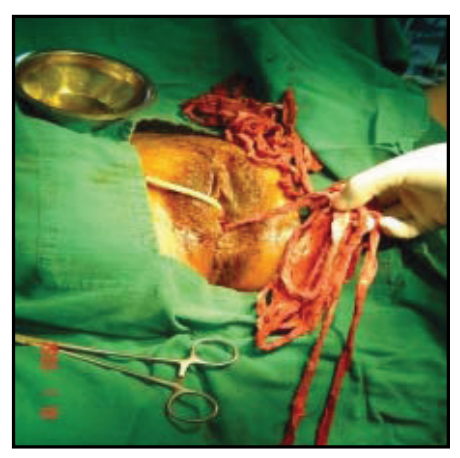

Fig. 4 (a): Uterine pack being removed 


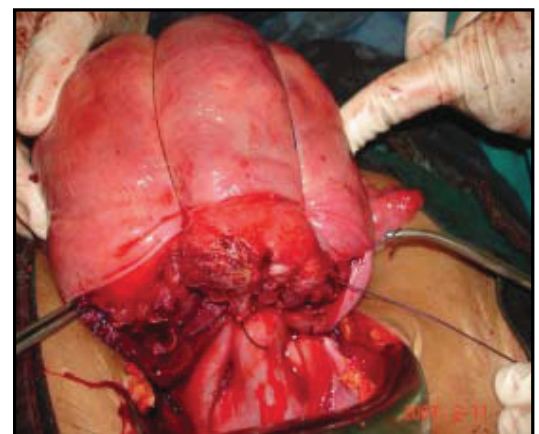

Fig. 4 (b): B-lynch suture

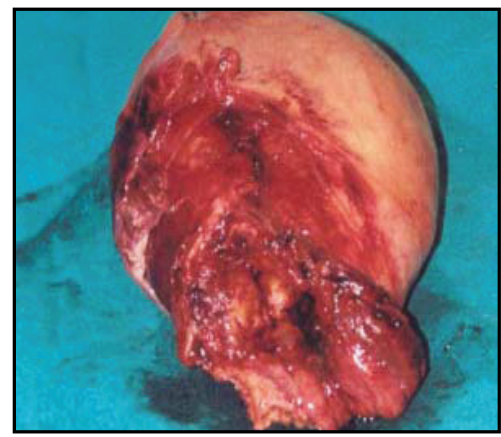

Fig. 5: Subtotal hysterectomy

A patient with primary CS for fetal distress had right sided salpingoophrectomy along with subtotal hysterectomy due to iatrogenic injury sustained while performing ovarian vessel ligation.

\section{Risk factors for hemoperitoneum and relaparotomy}

There were 6 cases of previous CS, of which one was previous $2 \mathrm{CS}$; one had marginal placenta previa, one underwent VBAC with vacuum delivery and the other with obstetric cholestasis with deranged LFT. There were 2 cases of placenta previa including the one with previous CS, 2 with eclampsia with its impending features and 4 each with nonprogress of labour (NPOL), prolong 2nd stage of labour and obstructed labour with hand prolapse.

\section{Maternal mortality}

There were 3 maternal mortalities. In the first case, though second intervention was repeated within 8 hours, death occurred because of lack of appreciation of blood loss which must have been more than estimated. She underwent subtotal hysterectomy but succumbed to DIC due to hematoma at bilateral uterine angles, extending to the anterior and posterior parietal wall with haemoperitoneum of more than1 liter.
The other mortality was a referred case with primary CS with gradual progression of abdominal distention on the first post operative day with pallor and no urinary output. A huge right sided broad ligament hematoma measuring about $15 \times 15 \mathrm{~cm}$ and extending towards the lumbar region was observed along with atonic uterus. It took 48 hours for second intervention where subtotal hysterectomy was carried out but succumbed to death.

The 3rd mortality was also a referred case where CS was done for non-progress of labour at $40+$ weeks and was referred to us for decreased urine output. She was severely pale and had abdominal distention with fluid thrill and shifting dullness. Urine output was minimal. $\mathrm{Hb}$ and platelet count were very low that delayed the reopening (16 hrs later) that also required blood products like fresh blood and PRP. On laparotomy, there was hemoperitoneum of more than a liter with multiple oozers in the left angle of the lower uterine segment. Bluish discoloration of peritoneum in the region of the $\mathrm{B} / \mathrm{L}$ broad ligament and vesicouterine fold of peritoneum was also observed. Oozers and the left angle were secured again. Despite of receiving multiple pints of fresh blood and PRP and with much longer stay in ICU for more than a month she succumbed to death due to complications like pulmonary oedema and multi organ failure.

\section{Associated morbidities}

In 5 subtotal hysterectomies associated morbidities were consumptive coagulopathy (2), gapped wound (1) wound infection (1) and ureteric fistula following ureteric ligation (1)

\section{Discussion}

Postoperative intraperitoneal as well as per vaginal hemorrhage in reality is a life threatening situation and has been tactfully combated by relaparotomies in obstetric surgeries as mentioned in two papers written a decade apart.3, 4 A series of 121 relaparotomies in 16,719 cases of laparotomy have depicted hemorrhage $(19 \%)$ as one of most important element amongst the others, such as ileus $(25 \%)$, peritonitis $(32 \%)$, wound rupture and burst abdomen $(22 \%)$, and other causes (2\%) 5 .

The paper dealt with all the obstetric cases that required second intervention (laparotomy) following primary CS. A total of 20 cases have been reviewed so far with an intention of carrying this study further. In about half of the cases $(11,61 \%)$ associated risk factors like previous CS $(6,30 \%)$, eclampsia $(2,11 \%)$ major degree and marginal placenta previa $(2,11 \%)$ with previous CS and, NPOL $(4,20 \%)$ along with obstructed labour were observed that could have been major contributors to relaparotomy. 
Detection was early and easier due the striking features of rising pallor with tachycardia ranging from 100-140 beats/ min, falling blood pressure, sweating, dizziness, abdominal distention and decreasing urinary output. Atony of uterus was seen in about 4 cases. Pervaginal bleeding either on the OT table while performing vaginal toileting or in the post operative ward were $6(30 \%)$ and the amount of blood loss was from $200-700 \mathrm{ml}$. These were further aided by USG and haemoperitoneum was diagnosed in $8(40 \%)$ cases, which made the diagnosis prompt and early intervention possible.

The time taken for second intervention varied from immediate 15 minutes like in cases who were reopened in the OT table following intense pervaginal bleeding and as late as 3 days as in a case referred from a zonal hospital. Late diagnosis after 24 hours were made in 5(28\%) cases mainly because of misleading normal vitals to begin with or a normalizing vitals after a brief period of circulatory collapse (unrecordable pulse and blood pressure) erroneous to an extent that a temporary improvements presumed after collapse taken for wellbeing cost lives in women following elective CS for CPD and emergency CS for NPOL in primi. This must have been produced from the infusion of crystalloids and colloids coping to replace the intraperitoneal blood loss with fair amount of hydration partially. Decreasing urine output which also signifies blood loss should also be taken into consideration, as had happened in one of the cases who was referred to us for the same. Therefore, early recognition of signs of intraperitoneal hemorrhage as well as vaginal bleeding are an important aspect, as these may not persist for long with illusive sense of recovery with fatal ends. Therefore, it is imperative to appreciate even the slightest of the hemodynamic changes so that early intervention can be introduced. Increasing abdominal girth $(9,45 \%)$ with shoulder tip pain as early as $12 \mathrm{hrs}$ of primary CS in a case of major placenta previa, were also ways of diagnosing intraperitoneal bleed that led to prompt intervention.

Laparotomy performed even with benefit of doubt during vaginal toileting in operating room after caesarean made a discovery of bleeders in the uterine placental bed which required multiple suture application as well as B-lynch compression suture. Similarly tears that had extended from the uterine incisions $(3,17 \%)$ were also repaired with multiple haemostatic sutures and B lynch application.

About a fourth cases $(5,28 \%)$ presented with extensive hematomas, including one with rectus sheath hematoma, where primary CS was done for deranged LFT with hypothyroidism with previous CS. There was fluctuation in her blood pressure at the time of surgery following spinal anesthesia and the systolic pressure dropping to $70 \mathrm{~mm}$ of $\mathrm{Hg}$. She was revived with atropine and mephentermine administration with further drop in the post operative period. After $28 \mathrm{hrs}$ due to gradual fall in the hemoglobin from 10 to 8 to $5 \mathrm{gm} \%$ and with the aid of USG suggesting hemoperitoneum, relaparotomy was performed to find rectus sheath hematoma with sippage of the blood into the peritoneal cavity causing haemoperitoneum. The blame could be place on to the falling blood pressure with subsequent rise in post operative period or to the deranged LFT. Broad ligament hematomas (4, 22.22\%) developed following primary surgery for CPD and fetal distress needed subtotal hysterectomy $(2,11.11 \%)$ but both these patients succumbed to death.

Though the controversy regarding the uterine packing for obstetric hemorrhage loom larger, it was found to be very useful in a case of CS performed for impending eclampsia, with failure of bilateral uterine, ovarian vessel ligation and B-lynch suture too.

Relaparotomy for obstetric haemorrhage has obtained a special benefit after receiving institution for pelvic packing where 10-12 dry pads are firmly packed once the cessation of bleeding is ensured after pressing over the alleged bleeding site for 5-10 minutes.6, 7 This has been applied at caesarean section for morbidly adherent anterior placenta previa over a previous CS scar during dire emergency from uncontrollable hemorrhage and related coagulopathy. 6 The packs were removed 36-48 hours later with good results.

It has been reported that relaparotomy for dehiscence and obstruction carried minimal risk whereas bleeding and infection entailed moderate risks and anastomotic leak had the highest mortality rate in 55 cases analyzed between 1996- 2000.8 There were mortality in two of the 16 cases. 9 We also had mortalities related to the primary presentation of the patient rather than to the relaparotomy.

As observed, almost all the cases except the one with obstetric cholestasis with previous CS, all other cases had to be reopened because of the human error. Thus to ensure that there will be no further reason to reopen once the primary surgery is over, few surgical principles should always be born in mind;

- Proper securing of the uterine incision and angles during CS, looking for extension both upward and downward. Securing the uterine edges and angular bleeding with figure of 8 sutures. There are suggestions to use square sutures and Heaney sutures, which are still not in practice.

- Ensure regaining of normal blood pressure (especially with regional anesthetic agents) before closure as this 
may obviate hematoma from small bleeders which were met in a case with $\mathrm{T}$ shaped extension and in a case with deranged LFT with previous CS, that were reinforced later.

- Whenever the doubt exits, to wash the peritoneal cavity by normal saline, which opens the bleeding capillaries by washing away the clots, which later can be coagulated.

- Putting an abdominal drain if there are venous oozers that have been left behind (not for the arterial bleeders) is always a wiser decision.

It is better to acknowledge and anticipate the probabilities of post operative intraperitoneal hemorrhage rather than treat them later, but at the same time tachycardia and hypotension which are the earliest signs along with decreasing urine output in these cases must be closely monitored and investigated so as to avoid morbidity and mortality related to late relaparotomy.

\section{Conclusion}

Choosing of the right technique, complete haemostasis and meticulous closure of all surgical incisions will prevent the need for laparotomy following LSCS. Vigilant monitoring of all the post operative patients will lead to early diagnosis of intraperitoneal / pervaginal bleeding and its management, thus preventing morbidity and mortality owing to late diagnosis

\section{References}

1. Duff P Sparks J, Hawley C.Extensive. An unusual complication of culdocentesis: intraperitoneal hemorrhage from uterine laceration. J Reprod Med 1983; 28(3): 221-2

2. Banas T, Boryczko M, Durzynska-Urbaniec J.Intraperitoneal hemorrhage due to the rupture of right ovarian artery in the second day of puerperium. Ginekol Pol 2004; 75(9): 729-32

3. Cereseto P1, Fernandez Jm. Gynecological relaparotomy. Dia Med.1962; 34: 771-2.

4. Tamas Z. Emergency relaparotomy after gynecologic and obstetric operations. Orv Hetil. 1972; 113 (33): 1969-71.

5. Tera H, Aberg C. Relaparotomy. A ten-year series. Acta Chir Scand. 1975; 141(7): 637-44

6. Ghourab S. Abdomino-pelvic packing to control severe hemorrhage following caesarean hysterectomy. J Obstet Gynaecol 1999; 19(2): 155-8
7. Holub Z, Feyereisl J, Kabelik L. Successful treatment of severe postpartum bleeding after caesarean section using recombinant activated factor VII. Rittstein T. Ceska Gynekol 2005 Mar; 70(2):144, 146-8.

8. Ching SS, Muralikrishnan VP, Whiteley GS. Relaparotomy: a five-year review of indications and outcome. Int J Clin Pract. 2003; 57(4): 333-7.

9. Mayer HG, Lirmberger M. Early abdominal second interventions following gynecologic surgery. Zentralbl Gynakol 1975; 97(11): 692-5 